\title{
In vitro osteoclast-like and osteoblast cells' response to electrospun calcium phosphate biphasic candidate scaffolds for bone tissue engineering
}

\author{
I. Wepener ${ }^{\mathrm{a}, \mathrm{b}, *}$, W. Richter ${ }^{\mathrm{a}}$, D. van Papendorp ${ }^{\mathrm{b}}$, A.M. Joubert ${ }^{\mathrm{b}}$ \\ Council for Scientific and Industrial Research, Polymers and Composites, PO Box 395, Pretoria, 0001, \\ South Africa \\ Department of Physiology, University of Pretoria, Pretoria, 0001, South Africa
}

\begin{abstract}
Successful long term bone replacement and repair remain a challenge today. Nanotechnology has makes it possible to alter materials' characteristics and therefore possibly improve on the material itself. In this study, biphasic (hydroxyapatite/ $\beta$-tricalcium phosphate (HA/ $\beta$-TCP)) nanobioceramic scaffolds were prepared by the electrospinning technique in order to mimic the extracellular matrix (ECM). Scaffolds were characterised by scanning electron microscopy (SEM) and Attentuated Total Reflectance Fourier Transform Infrared (ATRFTIR). Osteoblasts as well as monocytes that were differentiated into osteoclast-like cells, were cultured separately on the biphasic bioceramic scaffolds for up to 6 days and the proliferation, adhesion and cellular response were determined using lactate dehydrogenase (LDH) cytotoxicity assay, nucleus and cytoskeleton dynamics, analysis of the cell cycle progression, measurement of the mitochondrial membrane potential and the detection of phosphatidylserine expression. SEM analysis of the biphasic bioceramic scaffolds revealed nano fibers spun in a mesh-like scaffold. Results indicate that the biphasic bioceramic electrospun scaffolds are biocompatible and have no significant negative effects on either osteoblasts or osteoclast-like cells in vitro.
\end{abstract}

Keywords: electrospinning; hydroxyapatite; osteoblast; osteoclast-like; bone tissue engineering.

*Corresponding author. Address: Council for Scientific and Industrial Research, Polymers and Composites, PO Box 395, Pretoria, 0001, South Africa. Tel. +27(0)12 8413313 E-mail address: iwepener@csir.co.za (I. Wepener) 


\section{Introduction}

Conventionally, bone replacement and repair studies have always focussed on strong bioinert materials. Nowadays the focus has shifted towards materials that can mimic living tissue (biomimetic) and assist in the healing process (i.e. be replaced by natural bone): thus they are bioactive, as well as bioresorbable [1,2]. Bioactive materials have the ability to interact with the surrounding tissue by means of ion-exchange. This interaction gives rise to a biologically active carbonate apatite layer forming on the implant that is chemically equivalent to the mineral phase of bone $[3,4]$. When bioresorbable materials are implanted into the body they start to dissolve, whilst being replaced by tissue $[3,5,6]$.

Currently, the most widely used synthetic bioactive bone substitute is calcium phosphatebased materials. However, these calcium phosphate-based materials (i.e. hydroxyapatite (HA) and $\beta$-tricalcium phosphate ( $\beta$-TCP)) do not fulfil the requirements for bone repair and replacement. Calcium phosphates, although chemically similar to bone, lack collagen fibres for strength [2,7-9]. These substitutes are brittle and are not prone for use in load bearing circumstances. The general bioactivity of current substitutes should also be improved to promote faster and better bone reconstruction in the patient. An important fact to remember is that natural occurring HA crystals are nano-sized, while most widely used substitutes still have macro-sized grains $[4,7,10,11]$. Micro- and macro-porosities of the substitutes play a major role in their effect on biological properties. It has been shown that pore sizes around $260 \mu \mathrm{m}$ of the implant had the most successful in-growth. Pore interconnection sizes play an important role when hard and soft tissue in-growth is required and this lack of early vascularisation is a major drawback of most of the current bone tissue engineering techniques [3,11-13]. This is not always taken into account and affects current products on the market namely orthopaedic devices such as screws, plates, nails, and hip and knee replacements, dental cranio-maxillofacial products such as titanium (Ti) plates, sheets and screws and orthosis products such as artificial legs made from carbon composite materials [14]. These products, being metal, do not possess the adequate porosity for vascularisation and tissue ingrowth to occur.

The ideal biomaterial for bone replacement implanted into the body should be completely resorbed by osteoclasts over time, while osteoblastic activity deposits new mineralised bone at the site. The reason for this is that bone does not only function in a structural role, but is also a site for muscle anchoring and bone marrow production while playing a significant role in mineral homeostasis [15-17]. 
These dynamic functions can not be performed by "dead material" such as synthetic HA or $\beta$ TCP. Bone is dynamic living tissue, therefore it is important that novel bioceramics are developed that will initially function as bone replacement attending to all the requirements such as biocompatibility, structure, filler, as well as load-bearing. These bioceramics will also be able to activate the cellular response to recruit osteoclasts, osteoblasts and endothelial cells to the implant site for the resorbtion of the implant whilst enhancing the formation of new living bone $[2,7,8,18-20]$. Many different factors influence the activity of bone in the body which makes it crucial to understand the dynamics of bone and its interaction with the environment before real advances can be made in bioceramics for bone replacement and repair, as well as tissue engineering $[4,21,22]$.

Many studies have shown that HA is osteoconducting as well as osteoinducting. Surfaces containing HA, support osteoblastic cell adhesion, growth, differentiation and new bone formation [23,24]. Mesenchymal stem cells in surrounding tissues are stimulated and therefore osteoinduction occurs. Bone-forming osteoblast cells are then formed from these stimulated stem cells. Many studies have aimed to provide a more detailed understanding of the osteoinduction potential of HA and many of them have showed osteoinduction in various hosts including baboons [24-28].

In this study, electrospun biphasic (HA/ $\beta-\mathrm{TCP})$ nanobioceramic scaffolds were prepared and tested in vitro. Human osteoblasts (hFOB 1.19) and monocytes (THP-1), differentiated into osteoclast-like cells, were cultured on the bioceramic scaffolds, and the adhesion and cellular response were studied in vitro. Techniques included a cytotoxicity assay, nucleus and cytoskeleton dynamics, analysis of the cell cycle progression, measurement of the mitochondrial membrane potential and the detection of phosphatidylserine expression. Scaffolds were characterised by scanning electron microscopy (SEM), X-ray diffraction (XRD), and Attentuated Total Reflectance Fourier Transform Infrared (ATR-FTIR).

Unravelling signalling pathways, and the relationship between osteoclasts and osteoblasts when responding to an implanted biomimetic bone scaffold, will provide improved understanding of 'bioactive ceramic' surface features for biomineralisation, as well as the bone regeneration process. 


\section{Materials and methods}

\subsection{Materials}

HA powder was obtained from Merck (Darmstadt, Germany) and $\beta$-TCP powder from Fluka (Steinheim, Germany). Human fetal osteoblast cells (hFOB 1.19) and the human acute monocytic leukemia cell line (THP-1) was obtained from the American Type Culture Collection (ATCC, Arlington, VA, USA). Dulbecco's Modified Eagle's Medium (DMEM), Nutrient mixture F-12 (HAM), Roswell Park Memorial Institute (RPMI-1640) medium, fetal bovine serum (FBS), antibiotics and trypsin-EDTA were purchased from Gibco (Life Technologies, Johannesburg, South Africa). Annexin V-FITC kit, a MitoCapure $^{\mathrm{TM}}$ Mitochondrial apoptosis detection kit and a lactate dehydrogenase (LDH) cytotoxicity kit were purchased from BIOCOM biotech (Pty) Ltd. (Pretoria, South Africa) and manufactured by BioVision Inc. (Milpitas, California, USA). A cell counting kit, 1,25-dihydroxyvitamin $\mathrm{D}_{3}$ $\left(\mathrm{VD}_{3}\right)$, glacial acetic acid and acetone were purchased from Sigma-Aldrich SA (Pty) Ltd. (Johannesburg, South Africa).

\subsection{Fabrication of nano-biphasic scaffolds}

Electrospinning is used for the formation of electrostatic fibers. The technique uses electrical forces to produce fibers with diameters ranging from $2 \mathrm{~nm}$ to several micrometers [29,30]. An electrical field strength sufficient to overcome surface tension of the solution causes the droplets to elongate and eject very fine fibers. These fine fibers form non-woven mats when deposited.

HA powder (Merck, Darmstadt, Germany) and $\beta$-TCP powder (Fluka, Steinheim, Germany) was used during the production of the electrospun biphasic fibers. $40 \% \mathrm{HA}$ and $60 \%$ TCP ratio was used in a $30 \% \mathrm{w} / \mathrm{v}$ final total. Acetone $(50 \%)$ and acetic acid $(50 \%)$ was added to the ceramic powders while stirring vigorously for 1 hour. After 1 hour, gelatine was added drop wise to the mixture to reach 3, 5, 7 or $10 \%$ of total volume. This mixture was stirred for 30 minutes. The biphasic suspension was drawn into fibers upon the application of a high voltage $(15 \mathrm{kV})$. The fibers were collected on $22 \mathrm{~mm}$ round glass cover slips on an aluminium covered collector plate, spaced $15 \mathrm{~cm}$ from the needle tip. These biphasic bioceramic scaffolds were subsequently used for cell culture studies. 


\subsection{Characterisation of nano-biphasic scaffolds}

SEM was used to study the surface morphology of the biphasic bioceramic electrospun scaffolds at an accelerating voltage of $2 \mathrm{kV}$ after sputter coating with carbon. SEM analysis showed that nano fibers were formed. XRD showed that only HA and $\beta$-TCP was present in the electrospun scaffolds, however the input ratio of HA/ $\beta$-TCP decreased during electrospinning. ATR-FTIR spectroscopic analysis of electrospun biphasic scaffolds was performed on the PerkinElmer Spectrum 100 FT-IR Spectrometer (Shelton, Connecticut, USA).

\subsection{THP-1 cell culture}

The human monocyte-like cell line THP-1 (ATCC, TIB-202, Arlington, VA, USA) was used in this study since it can provide a continuous suspension culture with a relatively fast doubling time of 26 hours. The THP-1 cell line can also be co-cultured which could prove to be important for future studies.

THP-1 cells were cultured in RPMI-1640 medium, supplemented with $10 \%(\mathrm{v} / \mathrm{v})$ heatinactivated fetal calf serum and 1\% (v/v) penicillin/streptomycin (Gibco, Life Technologies, Johannesburg, South Africa) at $37^{\circ} \mathrm{C}$ in an atmosphere of $5 \% \mathrm{CO}_{2}$. Cells were grown as a suspension culture in $25 \mathrm{~cm}^{2}$ culture flasks and transferred to $75 \mathrm{~cm}^{2}$ culture flasks after 3 days. Before cell seeding, the ceramic samples were sterilised by immersion in $70 \%$ ethanol for 20 minutes, allowed to dry and placed in 6-well cell culture plates (Nunc, Roskilde, Denmark). The THP-1 cells were seeded on the biphasic bioceramic scaffolds (with $10 \%$ gelatine) at a density of 300000 cells/well in the presence of $10^{-7} \mathrm{M} \mathrm{VD}_{3} . \mathrm{VD}_{3}$ aids the differentiation of THP-1 monocytes into osteoclast-like adherent cells [31-34]. The adherent cells were cultured up to 6 days. Tissue culture plastic was used as control. THP-1 cells were seeded (50 000 cells/well) on 6-well culture plates (without any biphasic bioceramic scaffolds) under exact experimental conditions than the cells cultured on the biphasic bioceramic scaffolds. Suspended cells (before differentiation) were counted by the trypan blue assay using a haemocytometer under an optical microscope. Adherent cells (after differentiation) were detached from the surface by adding $0.05 \%$ trypsin containing $0.1 \%$ EDTA and counted in the same manner as the suspended cells. 


\section{5 hFOB 1.19 cell culture}

The human osteoblastic cell line hFOB 1.19 (ATCC, CRL-11372) differentiate into mature osteoblasts that express the normal osteoblast phenotype, ideal for studying differentiation, physiology, growth factor or other hormonal effects on the differentiation and function of osteoblasts.

hFOB 1.19 was cultured in DMEM and F12 media $(1: 1)$, supplemented with $10 \%(\mathrm{v} / \mathrm{v})$ heatinactivated fetal calf serum and 1\% (v/v) penicillin/streptomycin (Gibco, Life Technologies, Johannesburg, South Africa) at $37^{\circ} \mathrm{C}$ in an atmosphere of $5 \% \mathrm{CO}_{2}$. Cells were grown as an adhesion culture in $25 \mathrm{~cm}^{2}$ culture flasks and transferred to $75 \mathrm{~cm}^{2}$ culture flasks after 3 days. These cells were cultured to reach confluence. Before cell seeding, the ceramic samples were sterilised by immersion in 70\% ethanol for 20 minutes and placed in 6-well cell culture plates (Nunc, Roskilde, Denmark). hFOB 1.19 cells were seeded on biphasic bioceramic scaffolds (with $10 \%$ gelatine concentration) at a density of 150000 cells/well. The adherent cells were cultured up to 6 days with media changes every two days. Tissue culture plastic was used as control. hFOB 1.19 cells were seeded (50 000 cells/well) on 6-well culture plates (without any biphasic bioceramic scaffolds) under exact experimental conditions than the cells cultured on the biphasic bioceramic scaffolds. Adherent cells were detached from the surface by adding $0.05 \%$ trypsin containing $0.1 \%$ EDTA and counted by trypan blue assay using a haemocytometer.

\subsection{Cell toxcitiy: lactate dehydrogenase assay}

The interconversion of lactate and pyruvate is catalyzed by a cytosolic enzyme LDH that is soluble. When membrane integrity is compromised as a result from either apoptosis or necrosis, $\mathrm{LDH}$ is released from the cell. Therefore, LDH activity is used as an indicator of cell membrane integrity and is used to measure cytotoxicity that result from exposure of cells to chemical or foreign compounds (LDH-Cytotoxicity Assay Kit II, BioVision Incorporated, Milpitas, CA, USA).

Both cell lines were cultured as described in sections 2.4 and 2.5. After 6 days cells were trypsinized and resuspended in $1 \mathrm{ml}$ of culture medium. A background control (culture medium with no cells), low control (cells grown in wells without scaffolds) and high control (cells grown in wells with cell lysis solution included) according to the supplier's instructions, were included in this assay. Cells were centrifuged for five minutes to form a pellet and supernatant was discarded, and resuspended in $200 \mu \mathrm{l}$ culture medium. $10 \mu 1$ was transferred to an optically clear 96-well plate and $100 \mu 1$ of the LDH reaction mix (prepared according to 
the manufacturer's manual instructions) was added to each well. After 180 min incubation at room temperature the absorbance was read at $460 \mathrm{~nm}$ (reference wavelength of $630 \mathrm{~nm}$ ) with an $\mathrm{EL}_{\mathrm{x}} 800$ Universal Microplate Reader from Bio-Tek Instruments Inc. (Vermont, USA).

\subsection{Nucleus and cytoskeleton dynamics}

Blue fluorescent 4',6-diamidino-2-phenylindole (DAPI) and green fluorescent anti-rabbit IgG (H\&L)/DyLight ${ }^{\mathrm{TM}} 488$ conjugated (Thermo Fisher Scientific Inc) was used for DNA and cytoskeleton staining. After 6 days of cultivation the medium was removed and the cells were washed twice with PBS. Adherent cells were fixed with 3.7\% (v/v) paraformaldehyde for 10 min and permeabilised with $0.1 \%$ Triton X-100 (in PBS) for 10 min at room temperature. In order to detect the cytoskeleton, cells were incubated for 60 min with anti-rabbit $\operatorname{IgG}$ (H\&L)/DyLight ${ }^{\mathrm{TM}} 488$ conjugated (diluted 1:50) at room temperature followed by incubation with $1 \mu \mathrm{g} / \mathrm{ml}$ DAPI for $5 \mathrm{~min}$. Samples were washed again with PBS and mounted on glass slides in mounting medium Fluoromount-G (Olympus BX41, CC12 Soft imaging system, Hamburg, Germany).

\subsection{Cell cycle analysis}

Flow cytometry was used to analyse the cell cycle progression of THP-1 and hFOB 1.19 cells when grown on biphasic scaffolds. Propidium iodine (PI) was used to stain the nucleus to determine the amount of DNA present and the amount of PI fluorescence correlates with stages of the cell cycle during cell division. PI fluorescence (relative DNA content per cell) was measured using fluorescence activated cell sorting (FACS) FC500 System flow cytometer (Beckman Coulter SA (Pty) Ltd., Randburg, South Africa) equipped with an aircooled argon laser excited at $488 \mathrm{~nm}$. Data from at least 10000 cells/sample were analysed with CXP software (Beckman Coulter SA (Pty) Ltd., Randburg, South Africa). The data from particles smaller than apoptotic bodies and agglomeration of two or more cells were removed to improve accuracy. The non-commercially available Cyflogic software 1.2.1 (Pertu Therho, Turku, Finland) was employed to calculate cell cycle distributions by assigning relative DNA content per cell to sub- $\mathrm{G}_{1}, \mathrm{G}_{1}, \mathrm{~S}$ and $\mathrm{G}_{2} / \mathrm{M}$ fractions. The $\log$ forward scatter detector number 3 (FL3 Log), detects light emissions at $600 \mathrm{~nm}$ and PI molecules emit light at $617 \mathrm{~nm}$, therefore, the data obtained from this detector was represented as histograms on the $x$-axis.

Both cell lines were cultured as described in sections 2.4 and 2.5. [35,36]. After 6 days cells were trypsinized and resuspended in $1 \mathrm{ml}$ of culture medium. Upon termination, the supernatant of dead cells were also included in the assay. Cells were centrifuged for five minutes to form a pellet and supernatant was discarded. Thereafter, cells were resuspended in 
$200 \mu \mathrm{l}$ of ice-cold PBS containing 0.1\% FBS. $4 \mathrm{ml}$ ice-cold 70\% ethanol was added drop wise and cells were stored overnight at $4{ }^{\circ} \mathrm{C}$. Cells were pelleted by centrifugation at $300 \mathrm{xg}$ for $5 \mathrm{~min}$ and resuspended in $1 \mathrm{ml}$ of PBS containing PI $(40 \mu \mathrm{g} / \mathrm{ml})$ and incubated at $37^{\circ} \mathrm{C}$ for $45 \mathrm{~min}$ before analysis.

\subsection{Mitochondrial membrane potential measurement}

The intactness of the mitochondrial membrane was examined by the cationic dye, 5,5',6,6'tetrachloro-1,1'3,3'-tetraethylbenzimidazolylcarbocyanine iodide. When there is a reduction in the mitochondrial membrane potential, it is an indication of possible early apoptosis. The reduction of mitochondrial membrane potential is a result of the loss of electrochemical gradient across the mitochondrial membrane [37]. Quantitive apoptosis information is provided by the MitoCapture ${ }^{\mathrm{TM}}$ mitochondrial kit. Both cell lines were cultured as described in sections 2.4 and 2.5. After 6 days of growth on the scaffolds, cells were trypsinized and centrifuged at $13000 \mathrm{x}$ g. Upon termination, the supernatant was also included in the assay. The cell pellet was resuspended in $1 \mathrm{ml}$ of diluted MitoCapture solution $(1 \mu 1$ MitoCapture in $1 \mathrm{ml}$ pre-warmed incubation buffer) and incubated at $37^{\circ} \mathrm{C}, 5 \% \mathrm{CO}_{2}$ for $20 \mathrm{~min}$. After incubation cells were centrifuged at $500 \mathrm{x} g$ and the supernatant discarded. Cells were resuspended in $1 \mathrm{ml}$ of pre-warmed incubation buffer and immediately analysed. Analysis was conducted by means of FACS FC500 system flow cytometer (Beckman Coulter SA (Pty) Ltd., Randburg, South Africa). The FITC channel FL1 was used to detect apoptotic cells that showed diffused green fluorescence. Cyflogic version 1.2.1 software (Pertu Therho, Turko, Finland) was used to analyse data from at least 11000 cells.

\subsection{Detection of phosphatidylserine expression by annexin V-allophycocyanin staining}

The expression of the membrane phospholipid phosphatidylserine (PS) on the outside surface of the plasma membrane was analysed as a signal of apoptosis by staining with annexin $\mathrm{V}$, conjugated to a fluorochrome fluorescein isothiocyanate (FITC). Both cell lines were cultured as described in sections 2.4 and 2.5 and trypsinized after 6 days, (the supernatant was included in the assay); samples were centrifuged and resuspended in $1 \mathrm{ml}$ of $1 \mathrm{x}$ binding buffer and centrifuged at $300 \mathrm{x} g$ for $10 \mathrm{~min}$. The supernatant removed and the cells were washed and resuspended in $100 \mu 1$ of $1 \mathrm{x}$ binding buffer. Annexin V-FITC $(10 \mu 1)$ was added and the cells were incubated in the dark for $15 \mathrm{~min}$. After incubation, cells were washed with $1 \mathrm{ml} 1 \mathrm{x}$ binding buffer and centrifuged at $300 \mathrm{x} \mathrm{g}$ for $10 \mathrm{~min}$, discarding the supernatant and resuspending the cells in $500 \mu 1$ of $1 \mathrm{x}$ binding buffer solution. PI $(5 \mu 1$ of $40 \mu \mathrm{g} / \mathrm{ml})$ was added just before analysis to determine cell viability. PI and annexin V fluorescence was measured using FACS FC500 system flow cytometer (Beckman Coulter SA (Pty) Ltd., 
Randburg, South Africa) equipped with an air-cooled argon laser excited at $488 \mathrm{~nm}$. The data from at least 10000 cells were analysed with Cyflogic software 1.2.1 (Pertu Therho, Turko, Finland).

\subsection{Statistics}

Five different samples of each batch of electrospun scaffolds were analysed by SEM. A representative figure was chosen for each. Confocal studies were repeated twice and each experiment done in quintuplicate. Representative images were chosen for each experiment. Measurement of FITC-, PI- and 5,5',6,6'-tetrachloro-1,1'3,3'tetraethylbenzimidazolylcarbocyanine iodide -derived fluorescence was expressed as a ratio of the value measured for the cells grown on the electrospun scaffolds compared to cells grown on cell culture plate plastic (control) (mean relative fluorescence). Flow cytometry analysis involved data from at least 11000 events that was repeated thrice where after a representative figure was chosen for each experiment. The LDH assay was repeated thrice and data is expressed as intensity of absorbance and compared to the appropriate controls that were included. Data obtained from three independent experiments were shown as the mean \pm SD and were statistically analyzed for significance using the analysis of variance (ANOVA) single factor model followed by a two-tailed Student's $t$-test. Means were presented in bar charts, with T-bars referring to standard deviations. $P$-values $(0.05$ and less were regarded as statistically significant).

\section{Results and discussion}

ATR-FTIR was used to analyse the functional groups of the electrospun nanofibers (Fig. 1). Pure HA was analysed and the characteristic $\mathrm{OH}$-stretch was observed at $3570.8 \mathrm{~cm}^{-1}$ with a wide band at $3224 \mathrm{~cm}^{-1}$, which corresponds to the stretching mode of the O-H groups [38]. This can be attributed to absorbed water. These peaks are also exhibited by the electrospun scaffold, confirming the presence of HA after manufacturing. At $1019 \mathrm{~cm}^{-1}$, the stretching vibration for $\mathrm{PO}_{4}{ }^{3-}$ group from pure HA was obtained for the $\mathrm{P}-\mathrm{O}$ stretch, while in TCP it was obtained at $1000.3 \mathrm{~cm}^{-1}$. In this region $\left(800-1100 \mathrm{~cm}^{-1}\right)$, many peaks were observed in all three analyses. These peaks are characteristic to the phosphate ion oscillations modes, which are especially characteristic to highly crystalline HA. The peaks at $1416.2 \mathrm{~cm}^{-1}$ and $1486 \mathrm{~cm}^{-1}$ are possibly attributed to the carbonate ion $\mathrm{CO}_{3}{ }^{2-}$, which is an impurity present in both pure HA as well as the electrospun scaffold [30,39]. 


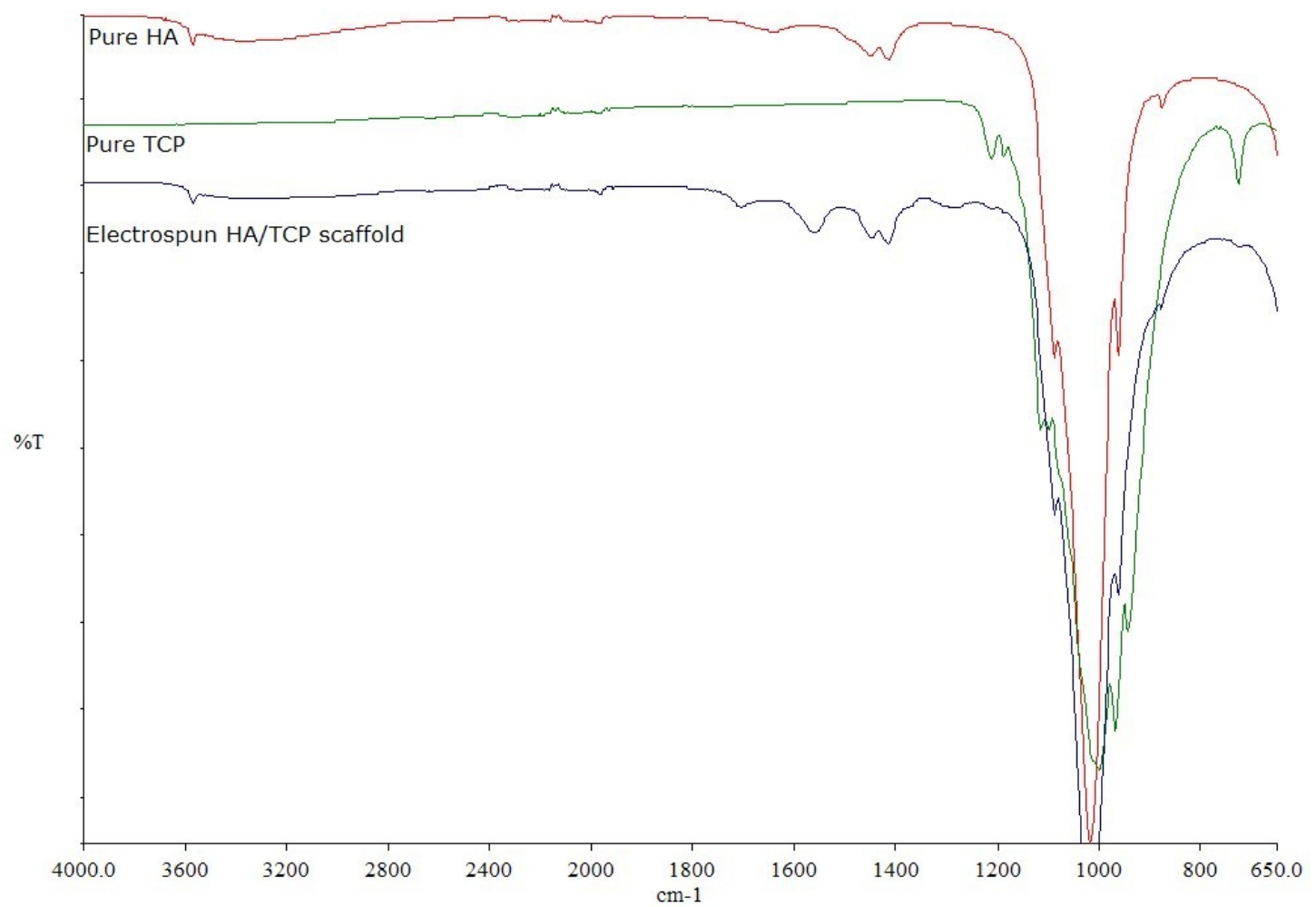

Fig. 1: ATR-FTIR analysis of (a) pure HA, (b) pure TCP and (c) electrospun HA/TCP scaffold.

The electrospinning technique is used to produce biomimetic nanofibers for tissue engineering applications since this technique can generate large surface area, interconnected pores, nanoscale dimensions and high porosity [40]. SEM images (Fig. 2) of electrospun biphasic scaffolds showed beadless and porous nanofibers that were formed under controlled conditions. The HA/ $\beta$-TCP with gelatine electrospun scaffolds showed uniform nano fibers and interconnected pores with fiber sizes in the $100 \pm 10 \mathrm{~nm}$ range. Scaffolds with 5, 7 and 10 $\%$ gelatine (Fig. $2 \mathrm{~b}-$ d) exhibited better uniformity of nano fibers when compared to scaffolds with only $3 \%$ gelatine (Fig. 2 a). At lower gelatine concentrations (Fig. 2 a \& b) some calcium phosphates can be seen embedded in the nano fibers which could be more desirable since it leads to an increase in surface roughness which is desirable for cell attachment. 


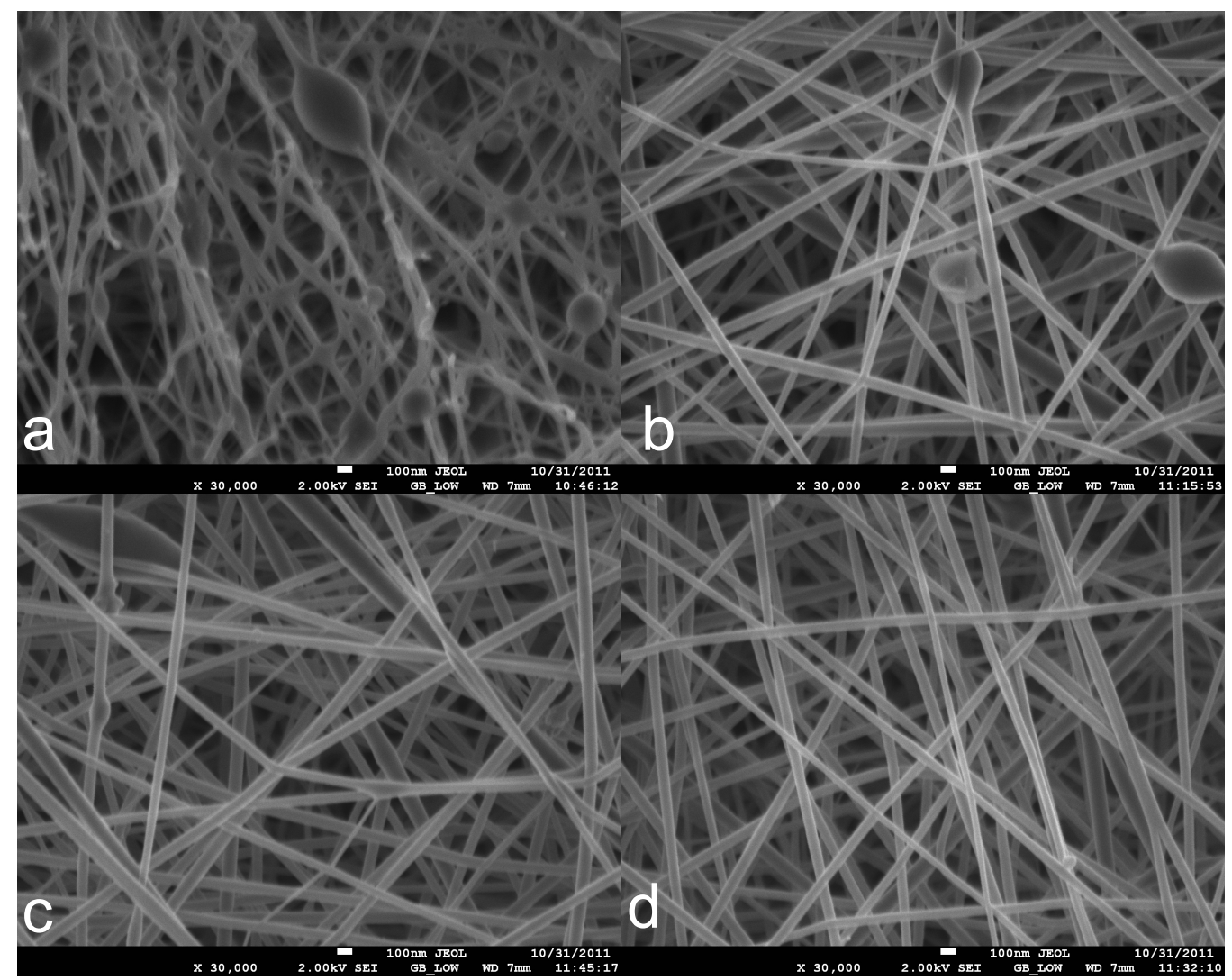

Fig. 2: Characterisation of the electrospun scaffolds by means of SEM. Images taken at 30 000x magnification of the electrospun scaffolds spun from 30\% (w/v) HA:TCP (40:60) with varying gelatin concentrations. (a) $3 \%$ (b) $5 \%$ (c) $7 \%$ (d) $10 \%$ (which was used in this study).

\subsection{Cell-scaffold interaction}

Intracellular responses, cell growth and cell morphology are greatly influenced by scaffold properties. In this study the effect of scaffold composition was analysed through cell viability, mitochondrial membrane potential, cell cycle and early apoptosis detection. Results from the LDH release study (Fig. 3) revealed that cells grown on the electrospun biphasic bioceramic scaffolds did not exhibit toxicity, even when grown for 21 days (results not shown). A slight increase in toxicity was seen over time, however, it was not significant to attribute it to the scaffolds. 

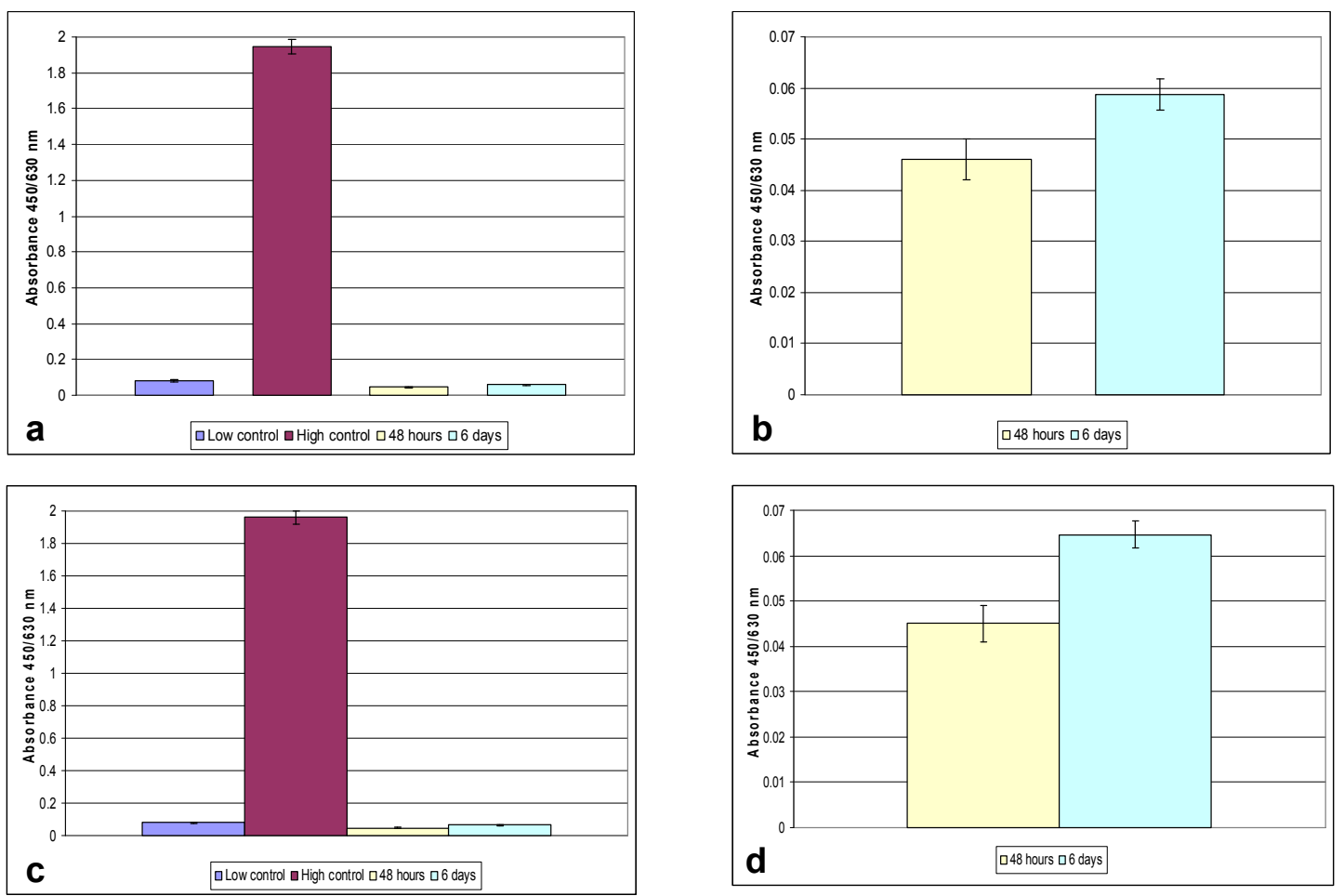

Fig. 3: Analysis of $\mathrm{LDH}$ release in osteoclast-like cells (a \& b) and osteoblast cells (c \& d) grown on electrospun scaffolds after 48 hours and 6 days with controls included (a \& c) and (b \& d) showing the samples.

Confocal microscopy of the stained cells (blue DAPI for nucleus staining and green fluorescent anti-rabbit $\mathrm{IgG}(\mathrm{H} \& \mathrm{~L}) /$ DyLight $^{\mathrm{TM}} 488$ conjugated for microtubulin staining) showed healthy, intact cells with uncompromised nuclei and microtubulin when osteoclastlike (Fig. 4 (b)) and osteoblast cells (Fig. 4 (d)) were grown on the biphasic electrospun scaffolds compared to the controls (culture plate) (Fig. 4 (a \& c)). Morphology of the THP-1 and hFOB 1.19 cells were not compromised when grown on the electrospun scaffolds. 

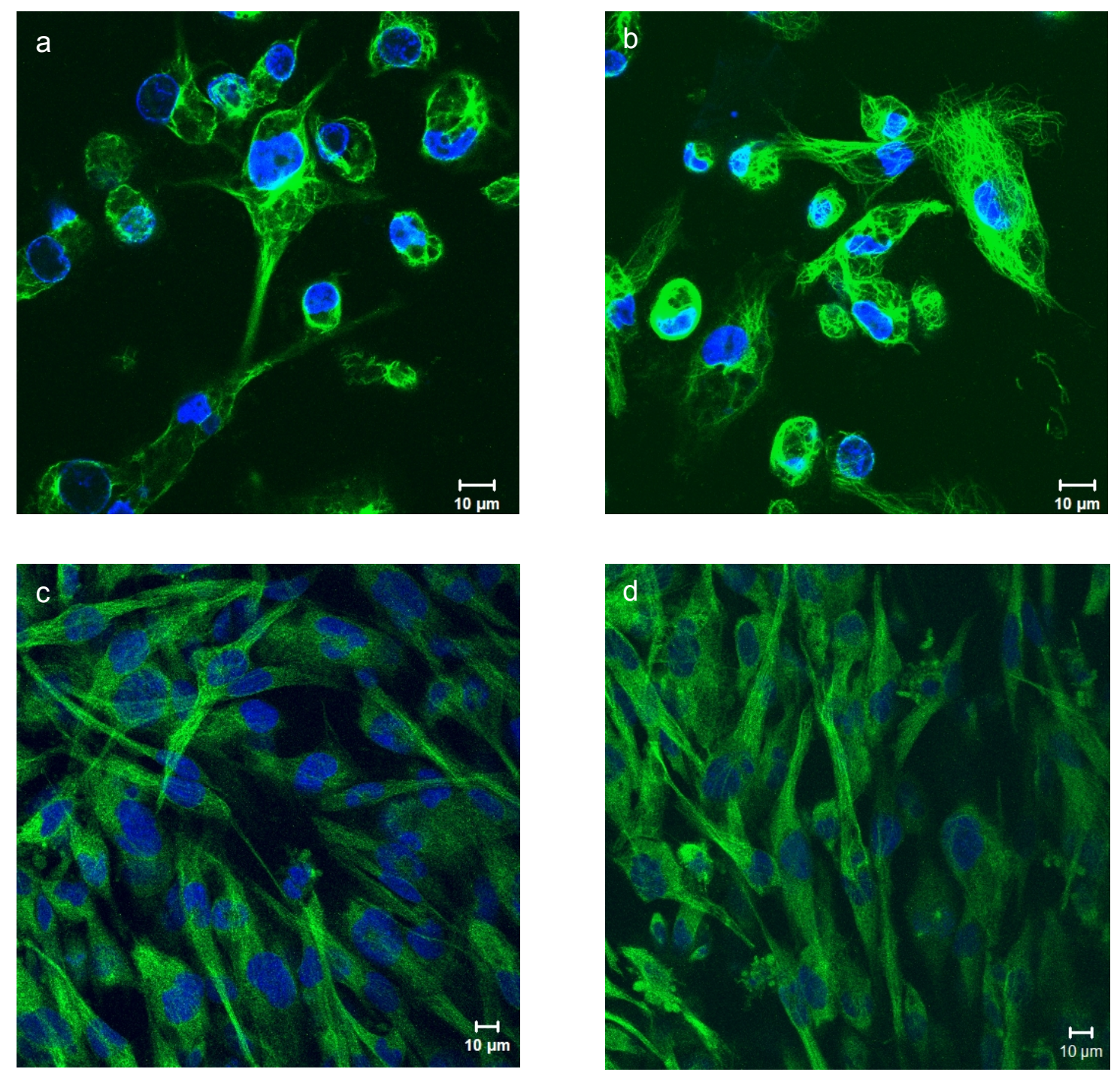

Fig. 4: Nucleus and cytoskeleton dynamics: Confocal microscopy images, utilizing blue fluorescent DAPI to stain the nucleus and green fluorescent anti-rabbit IgG $(\mathrm{H} \& \mathrm{~L}) / \mathrm{DyLight}{ }^{\mathrm{TM}}$ 488 conjugate staining tubulin structures of osteoclast-like cells (a \& b) and osteoblast cells (c \& d) grown on cell culture plate (control) (a \& c) and on electrospun scaffolds (b \& d).

Cell cycle analysis of the osteoclast-like cells grown on electrospun scaffold (Fig. 5 (b)), compared well to the control (culture plate) (Fig. 5 (a)). In both cases most cells were actively dividing in the $\mathrm{G}_{1}$-phase. In Fig. 5 (b) a slight increase in the sub-G $\mathrm{G}_{1}$ phase can be seen when compared to the control. This is expected when cells are growing on a scaffold and is characteristic of apoptotic cells. 


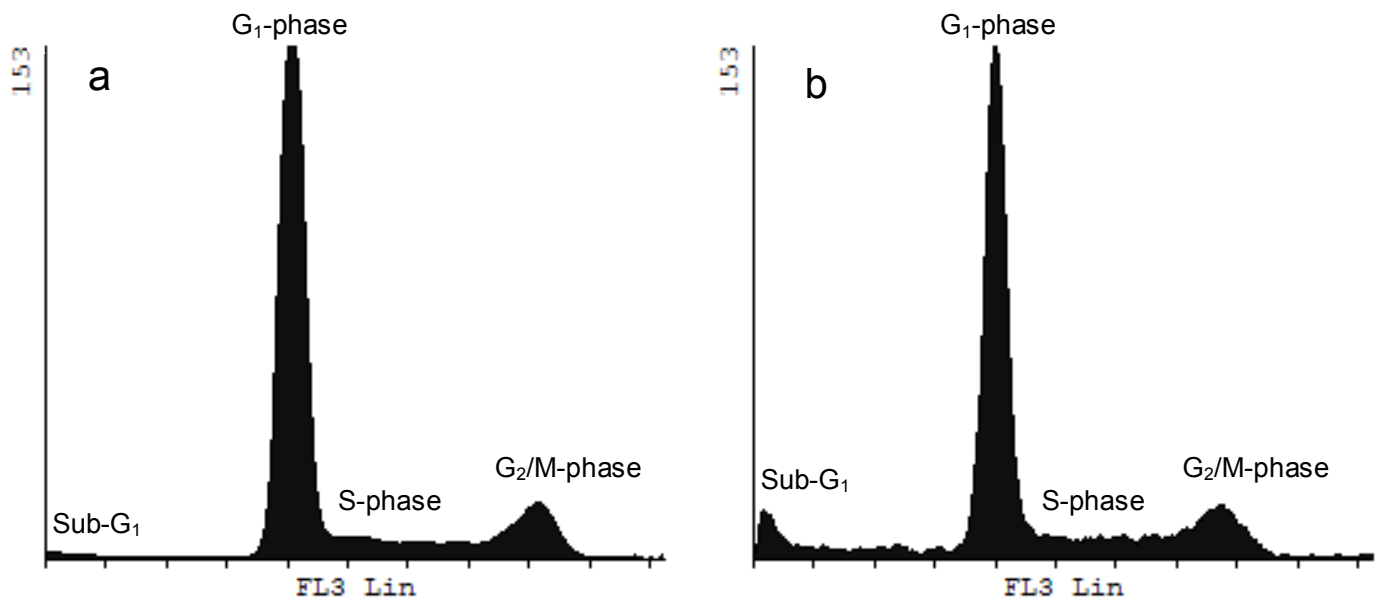

Fig. 5: Histogram representation of osteoclast-like cells grown for 6 days on (a) tissue culture plates (control) and (b) electrospun scaffold. Normal cell phase distribution is seen. Most cells are actively dividing in the $G_{1}$-phase. An increase in the sub- $G_{1}$ phase is observed in (b), from $1.72 \%$ in the control to $\mathbf{1 . 4 8 \%}$ in the sample grown on scaffolds. Significantly more cells were actively growing in the $G_{1}$-phase when compared to the control, however a decrease of cell percentage is seen in the S-phase when compared to the control. Figure 5 a and b show the different cell cycle phases as a representative of three repetitive experiments. In each sample at least 11000 events were counted.

Table 1: Cell percentages in the various cell cycle phases of osteoclast-like cells cultured on a control surface as well as on the electrospun scaffold.

\begin{tabular}{|l|l|l|l|l|}
\hline Surface & sub-G & $\mathrm{G}_{1}$ & $\mathrm{~S}$ & $\mathrm{G}_{2} / \mathrm{M}$ \\
\hline Control & $1.72 \pm 0.23$ & $62.05 \pm 1.10$ & $15.84 \pm 0.10^{*}$ & $12.88 \pm 0.75$ \\
\hline Electrospun scaffold & $1.48 \pm 0.29$ & $70.84 \pm 2.88^{*}$ & $9.42 \pm 0.60$ & $11.51 \pm 0.52$ \\
\hline
\end{tabular}
$* p<0.05$

Osteoblast cells' cycle were also analysed. Cells cultured on the electrospun scaffolds (Fig. 6 (b)) compared well to the control (Fig. 6 (a)). Most cells were actively dividing in the $\mathrm{G}_{1^{-}}$ phase. An increased number of cells grown on the scaffolds as well as on the control sample were observed in the $\mathrm{G}_{2} / \mathrm{M}$-phase. Cells growing on the electrospun scaffolds exhibited an increase in the sub- $G_{1}$ phase; once again this is expected when cells are cultured on scaffolds and is characteristic of apoptotic cells. Since a slight increase in the sub- $\mathrm{G}_{1}$ fraction was observed in this study in both the osteoclast and osteoblast cell cycle analysis, other assays analysing apoptosis and cell death were also employed. 

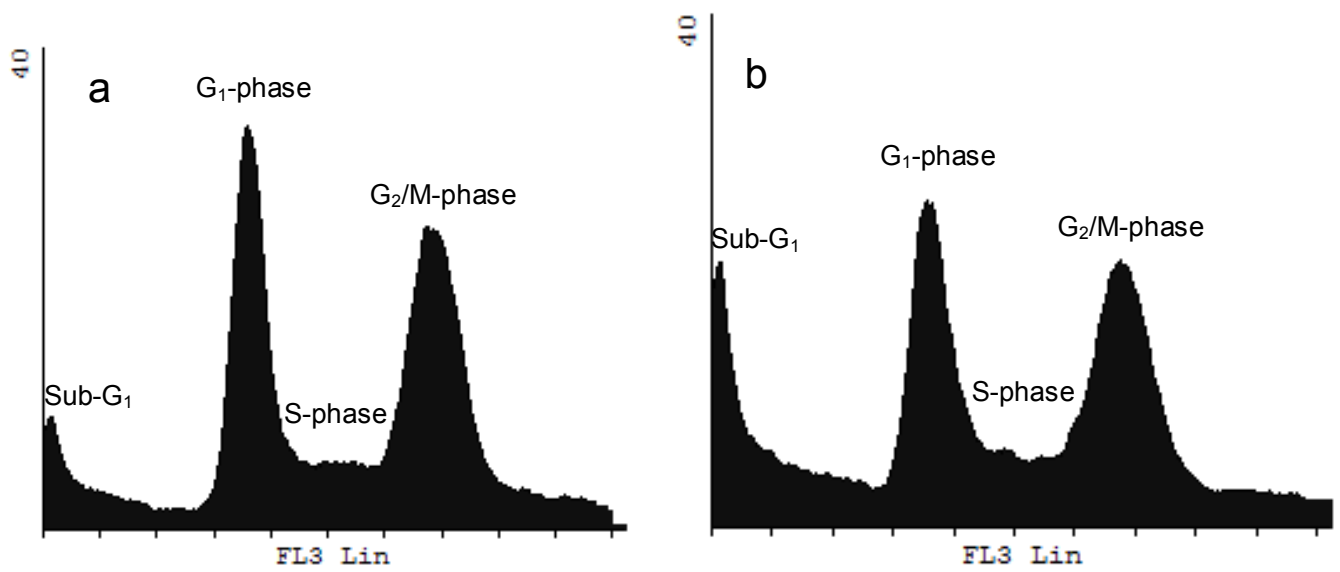

Fig. 6: Histogram representation of osteoblast cells grown for 6 days on (a) tissue culture plates (control) and (b) elestrospun scaffold. Normal distribution of the cell phases is detected, with an increase in the sub- $G_{1}$ fraction of the cells grown on the scaffold $(5.62 \%)$ when compared to the control (5.23\%), however not significant. There is a significant decrease in the percentage cells in the S-phase on the electrospun scaffold when compared to the control. Figure 6 a and b show the different cell cycle phases as a representative of three repetitive experiments. In each sample at least 11000 events were counted.

Table 2: Cell percentages in the various cell cycle phases of osteoblast cells cultured on a control surface as well as on the electrospun scaffold.

\begin{tabular}{|l|l|l|l|l|}
\hline Surface & sub- $\mathrm{G}_{1}$ & $\mathrm{G}_{1}$ & $\mathrm{~S}$ & $\mathrm{G}_{2} / \mathrm{M}$ \\
\hline Control & $5.23 \pm 0.49$ & $19.63 \pm 0.70$ & $9.52 \pm 0.55$ & $22.01 \pm 1.46$ \\
\hline Electrospun scaffold & $5.62 \pm 0.23$ & $19.11 \pm 1.12$ & $7.28 \pm 0.36^{*}$ & $18.26 \pm 1.43$ \\
\hline
\end{tabular}
$* p<0.05$

Mitochondria play a big role in programmed cell death. When the mitochondrial potential decreases, it is an indicator of early apoptosis. The mitochondrial membrane potential was measured by means of flow cytometry of the osteoclast-like cells. It revealed a slight increase in viable cells when grown on electrospun scaffolds (Fig. 7 (b)) when compared to the control (culture plates) (Fig. 7 (a)). This result indicated that the increase in the sub- $\mathrm{G}_{1}$ fraction during cell cycle analysis (Fig. 5 (b)) was probably not due to apoptosis and the cells would most likely return to an actively growing state. 


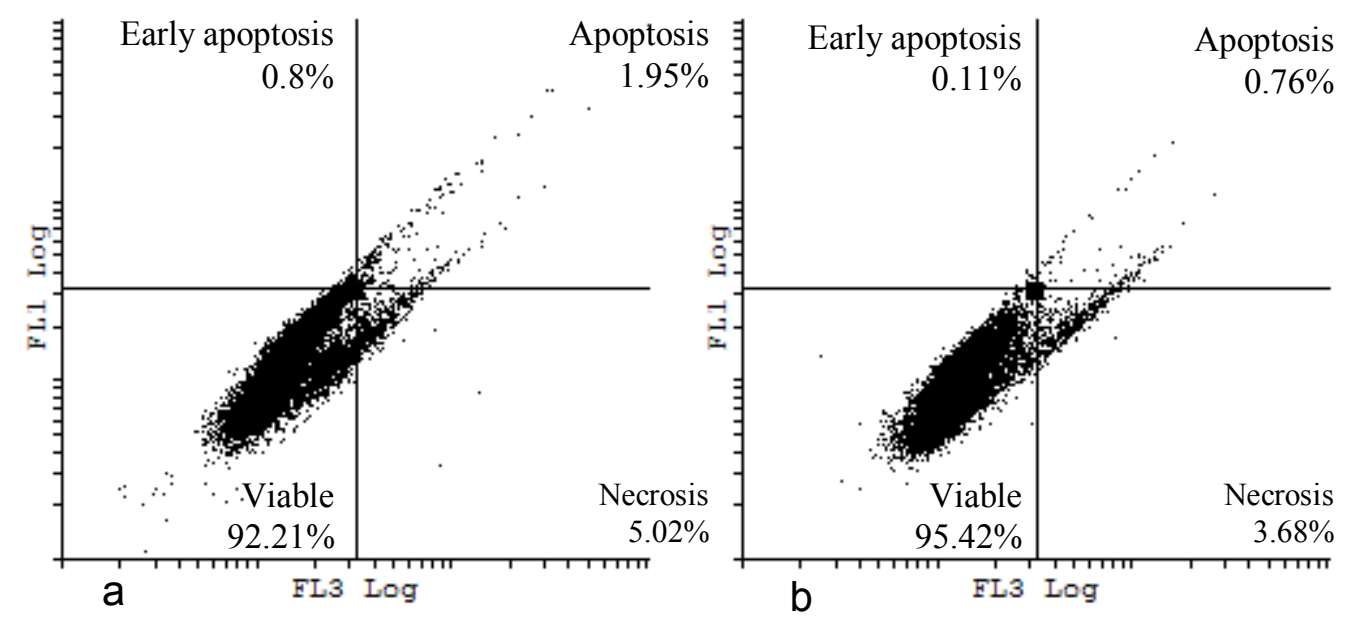

Fig. 7: Mitochondrial membrane potential measurement of osteoclast-like cells grown on (a) tissue culture plates (control) and (b) on electrospun scaffolds. Cells grown on electrospun scaffolds (b) exhibited a higher percentage of viable cells when compared to the control (a). Each figure is representative of three repetitive experiments where at least 11000 cells were counted.

Flow cytometry analyses revealed statistically insignificant decrease in the mitochondrial membrane potential of the osteoblast (hFOB 1.19) cells grown on the biphasic electrospun scaffolds (Fig. 8 (b)) when compared to the control (culture plate) (Fig. 8 (a)). Therefore, the increase in the sub- $\mathrm{G}_{1}$ fraction observed during cell cycle analysis (Fig. 6 (b)) can probably not be attributed to apoptosis. Results of mitochondrial membrane potential measurement revealed that no significant changes occurred in either cell line when the cells are grown on the electrospun biphasic bioceramic scaffolds.
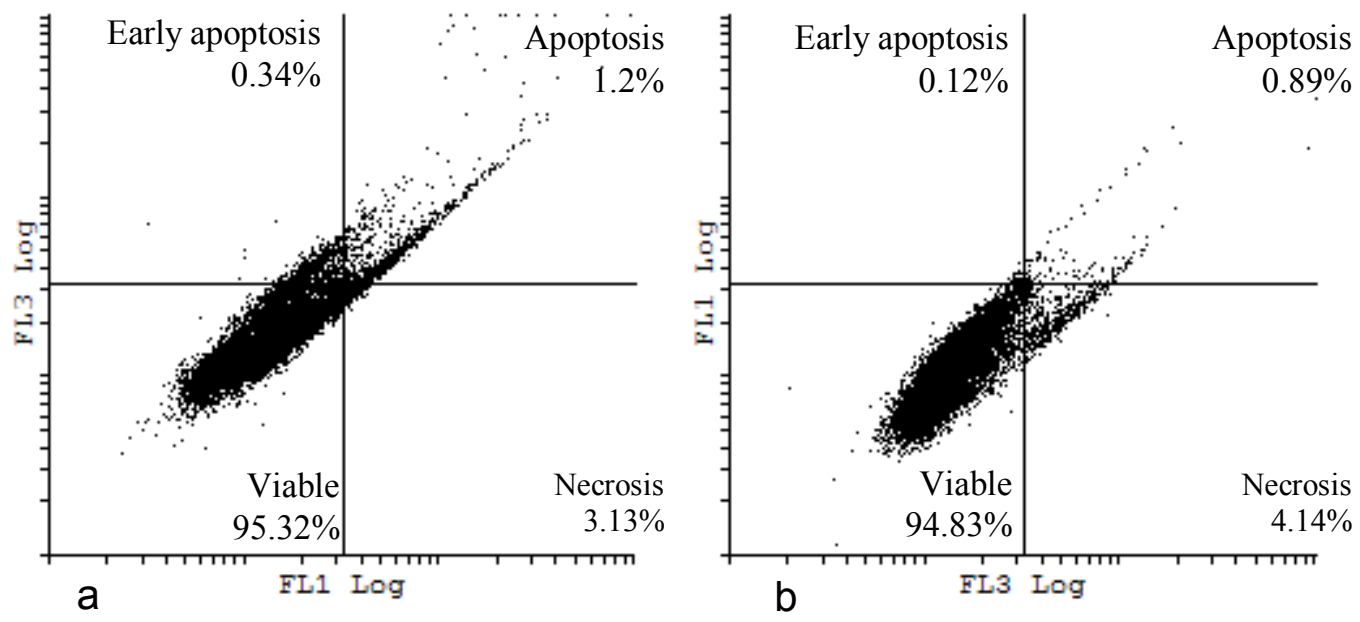

Fig. 8: Mitochondrial membrane measurement of osteoblast cells grown on (a) tissue culture plates (control) and (b) on electrospun scaffolds. Cells grown on electrospun scaffolds did not exhibit a significant decrease in viable cells and compared well to the control (a). Each figure is representative of three repetitive experiments where at least 11000 cells were counted. 
Flow cytometry was used to measure the induction of cell death by employing Annexin VFITC. Annexin V binds with strong affinity to phosphatidylserine that gets exposed on the outer surface membrane of cells when they enter early apoptosis. After 6 days of culturing, the osteoclast-like cells grown on the biphasic electrospun scaffold (Fig. 9 (b)) had $92.56 \%$ viable cells, almost exactly the same as the control with 93.08 \% (Fig. 9 (a)). Cells grown on the scaffold showed an increase in necrosis (4.23\%), but reduction in early (2.52\%) and late (1.58 \%) apoptosis (Fig. 9 (b)) when compared to the control (culture plate) (Fig. 9 (a)).
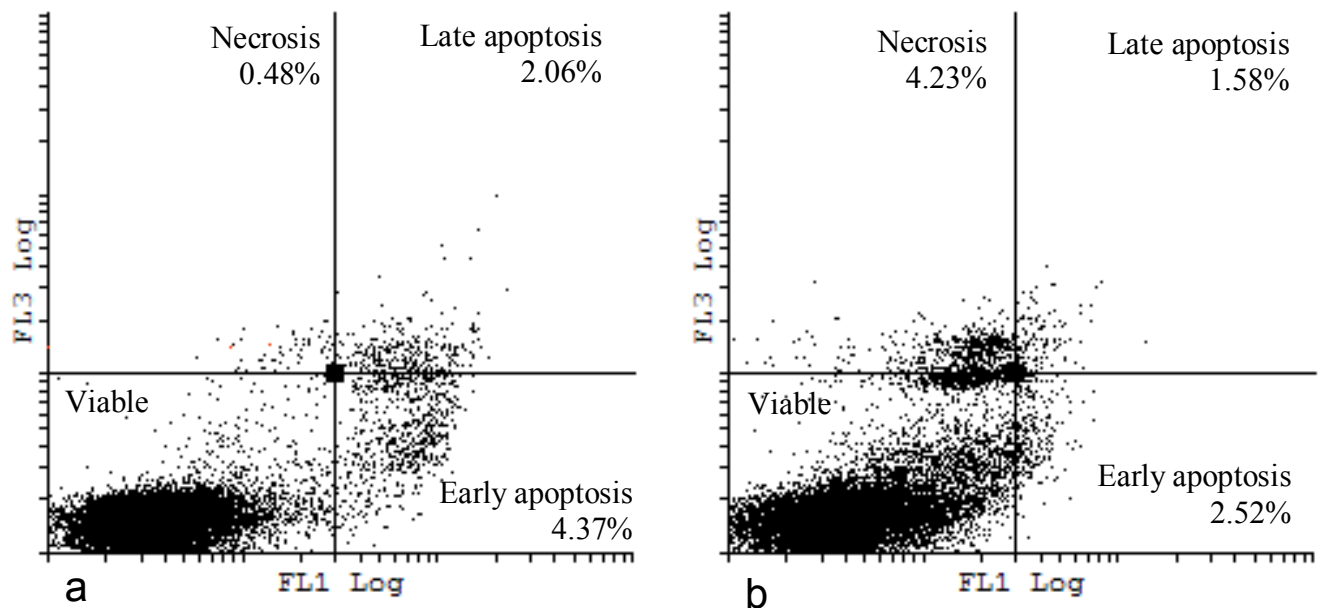

Fig. 9: Apoptosis detection through flow cytometry and annexin V-FITC of osteoclast-like cells (a) control (cells grown on cell culture plate only) and (b) cells grown on electrospun scaffolds. Propidium iodide (FL3 Log) versus annexin V-FITC (FL1 Log) dot-plots of cells. Cells grown on electrospun scaffolds (b) did not exhibit a decrease in viable cells and compared well to the control (a), however, an increase in necrosis was seen when cells were grown on the electrospun scaffolds, while a decrease in cell percentages was seen in the early apoptotic and late apoptotic phases of the cells grown on the electrospun scaffolds, when compared to the control. In each sample at least 11000 cells were analysed and each figure represents at least three repetitive experiments.

Table 3: Cell percentages of osteoclast-like cells grown on a control surface (tissue culture plastic) and on electrospun scaffolds, analyzing the different steps of apoptosis.

\begin{tabular}{|l|l|l|l|l|}
\hline Surface & Viable & Early apoptotic & Late apoptotic & Necrotic \\
\hline Control & $93.08 \pm 0.23$ & $4.37 \pm 0.14$ & $2.06 \pm 0.21$ & $0.48 \pm 0.05$ \\
\hline Electrospun scaffold & $92.56 \pm 0.81$ & $2.52 \pm 0.36^{*}$ & $1.58 \pm 0.18^{*}$ & $4.23 \pm 0.28^{*}$ \\
\hline
\end{tabular}
${ }^{*} p<0.05$

Osteoblast cells were grown for 6 days on the biphasic electrospun scaffold (Fig. 10 (b)), and on the control (culture plate) (Fig. 10 (a)). In both cases over $97 \%$ of the cells were viable and the difference in early apoptotic cells between cells grown on the control (culture plate) and the electrospun biphasic scaffold was negligible. 

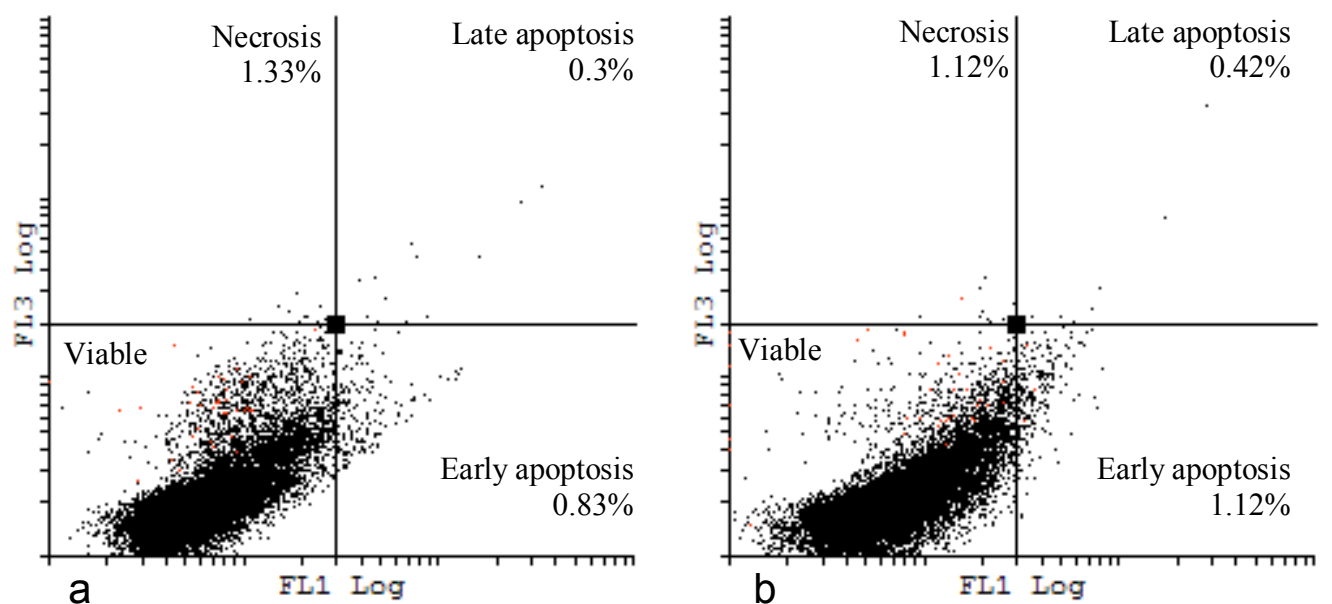

Fig. 10: Apoptosis detection through flow cytometry and annexin V-FITC of osteoblast cells (a) control (cells grown on cell culture plate only) and (b) cells grown on electrospun scaffolds. Propidium iodide (FL3 Log) versus annexin V-FITC (FL1 Log) dot-plots of cells. Cells grown on electrospun scaffolds (b) did not exhibit significant decrease in viable cells and compared well to the control (a). In each sample at least 11000 cells were analysed and each figure represents at least three repetitive experiments.

Table 4: Cell percentages of osteoclast-like cells grown on a control surface (tissue culture plastic) and on electrospun scaffolds, analyzing the different steps of apoptosis.

\begin{tabular}{|l|l|l|l|l|}
\hline Surface & Viable & Early apoptotic & Late apoptotic & Necrotic \\
\hline Control & $97.54 \pm 1.18$ & $0.83 \pm 0.66$ & $0.3 \pm 0.23$ & $1.33 \pm 0.36$ \\
\hline Electrospun scaffold & $97.33 \pm 0.62$ & $1.12 \pm 0.83$ & $0.42 \pm 0.06$ & $1.12 \pm 0.64$ \\
\hline
\end{tabular}
$* p<0.05$

\section{Conclusion}

Electrospinning proved to be an effective technique to create biphasic (HA/ $\beta-\mathrm{TCP})$ nanoscaffolds with uniform fibers and interconnected pores for cell growth and attachment. In this study a higher percentage of the calcium phosphates were used to produce the electrospun scaffold with a low gelatine percentage. In previous studies other researchers manufactured scaffolds with a 1:1 ratio of collagen to HA by electrospinning [41], $\beta$-TCP in gelatine at 5 , 10 and 20 wt.\% by cross-linking treatment [40], polymer-only scaffolds by electrospinning (PCL, chitosan, PCL/chitosan), as well as combining the polymers with HA [42]. In many instances the focus has been on biodegradable polymers only or combining a high percentage of polymer with a fraction HA to manufacture scaffolds. In this study it was possible to make use of two of the most abundant components of bone (HA and $\beta$-TCP), combining it with gelatine and manufacturing an electrospun biphasic scaffold. Osteoclast-like and osteoblast cells were cultured on the newly manufactured electrospsun biphasic scaffolds to monitor cell growth and cellular response to the scaffolds. No cytotoxicity was detected in both cell lines, while cells grown on electrospun scaffolds compared well to the control (culture plate) in all instances. Cells responded very well to electrospun biphasic scaffolds and did not show 
significant increases in apoptosis or a difference in cell morphology. From this data, it can be concluded that the electrospun biphasic scaffolds are biocompatible with and appropriate for adherence and growth of osteoclast-like and osteoblast cells for bone tissue engineering. Future co-culturing studies could prove useful to examine osteoclast and osteoblast interaction.

\section{Acknowledgements}

This study was financially supported by the Council for Scientific and Industrial Research (CSIR), Pretoria, South Africa. SEM analysis was performed at The National Centre for Nano-Structured Materials, CSIR, Pretoria, South Africa. Flow cytometric analysis was conducted at the Department of Pharmacology, Faculty of Health Sciences, University of Pretoria, Pretoria, South Africa. Special thanks to the people working in Prof A. Joubert's laboratory, Department of Physiology, Faculty of Health Sciences, University of Pretoria, Pretoria, South Africa for support.

\section{References}

1. Heinemann S, Heinemann C, Bernhardt R, Reinstorf A, Nies B, Meyer M, Worch H, et al. Bioactive silica-collagen composite xerogels modified by calcium phosphate phases with adjustable mechanical properties for bone replacement. Acta Biomater 2009;5:1979-1990.

2. Schilling AF, Linhart W, Filke S, Gebauer M, Schinke T, Rueger JM, Amling M. Resorbability of bone substitute biomaterials by human osteoclasts. Biomaterials 2004;25:3963-3972.

3. Heness G, Ben-Nissan B. Innovative bioceramics. Materials Forum 2004;27:104-114.

4. Pan H, Zhao X, Darvell BW, Lu WW. Apatite-formation ability - Predictor of “bioactivity”? Acta Biomater 2010;6:4181-4188.

5. Armentano I, Dottori M, Fortunati E, Mattioli S, Kenny JM. Biodegradable polymer matrix nanocomposites for tissue engineering: A review. Polym Degrad Stab 2010;95:2126-2146. 
6. Dorozhkin SV. Bioceramics of calcium orthophosphates. Biomaterials 2010;31:1465-1485.

7. Chevalier J, Gremillard L. Ceramics for medical applications: A picture for the next 20 years. J Eur Ceram Soc 2009;29:1245-1255.

8. Dorozhkin SV. Bioceramics based on calcium orthophosphates (Review). Glass \& Ceram 2007;64:442-447.

9. Sergey V. D. Biphasic, triphasic and multiphasic calcium orthophosphates. Acta Biomater 2012;8:963-977.

10. Von der Mark K, Park J, Schmuki P. Nanoscale engineering of biomimetic surfaces: cues from the extracellular matrix. Cell Tissue Res 2010;339:131-153.

11. Holzwarth JM, Ma PX. Biomimetic nanofibrous scaffolds for bone tissue engineering. Biomaterials 2011;32:9622-9629.

12. Wei J, Jia J, Wu F, Wei S, Zhou H, Zhang H, Shin J, et al. Hierarchically microporous/macroporous scaffold of magnesium-calcium phosphate for bone tissue regeneration. Biomaterials 2010;31:1260-1269.

13. Dorozhkin SV. Nanosized and nanocrystalline calcium orthophosphates. Acta Biomater 2010;6:715-734.

14. Hieu LC, Quoc LH, Thanh VV, Nguyen TD, An PV, Hung LT, Khanh L. Current medical product development for diagnosis, surgical planning and treatment in the areas of Neurosurgery, Orthopeadic and Dental-Cranio-Maxillofacial surgery in Vietnam. $2010 ;: 122-125$.

15. Patterson J, Martino MM, Hubbell JA. Biomimetic materials in tissue engineering. Materials Today 2010;13:14-22. 
16. Vallet-Regí M. Evolution of bioceramics within the field of biomaterials. C R Chim 2010;13:174-185.

17. Hench LL, Thompson I. Twenty-first century challenges for biomaterials J R Soc Interface 2010;7:S379-S391.

18. Schilling AF, Filke S, Brink S, Korbmacher H, Amling M, Rueger JM. Osteoclasts and Biomaterials. Europ J Trauma 2006;32:107-113.

19. Jones JR. New trends in bioactive scaffolds: The importance of nanostructure. J Eur Ceram Soc 2009;29:1275-1281.

20. Athanasou NA. The osteoclast-what's new? Skelet Radiol 2011;40:1137-1140.

21. Bohner M. Resorbable biomaterials as bone graft substitutes. Materials Today 2010;13:24-30.

22. Costa-Rodrigues J, Fernandes A, Lopes MA, Fernandes MH. Hydroxyapatite surface roughness: Complex modulation of the osteoclastogenesis of human precursor cells. Acta Biomater 2012;8:1137-1145.

23. Albrektsson T, Johansson C. Osteoinduction, osteoconduction and osseointegration. Eur Spine J 2002;10:S96-S101.

24. Habibovic P, Gbureck U, Doillon CJ, Bassett DC, van Blitterswijk CA, Barralet JE. Osteoconduction and osteoinduction of low-temperature 3D printed bioceramic implants. Biomaterials 2008;29:944-953.

25. Yuan H, Kurashina K, de Bruijn JD, Li Y, de Groot K, Zhang X. A preliminary study on osteoinduction of two kinds of calcium phosphate ceramics. Biomaterials 1999;20:1799-1806. 
26. Ripamonti U, Roden LC, Renton LF. Osteoinductive hydroxyapatite-coated titanium implants. Biomaterials 2012;33:3813-3823.

27. Ripamonti U, Crooks J, Khoali L, Roden L. The induction of bone formation by coralderived calcium carbonate/hydroxyapatite constructs. Biomaterials 2009;30:1428-1439.

28. Ripamonti U, Richter PW, Nilen RWN, Renton L. The induction of bone formation by smart biphasic hydroxyapatite tricalcium phosphate biomimetic matrices in the non-human primate Papio ursinus. J Cell Mol Med 2008;12:1-15.

29. Bhardwaj N, Kundu SC. Electrospinning: A fascinating fiber fabrication technique. Biotechnol Adv 2010;28:325-347.

30. Franco PQ, João CFC, Silva JC, Borges JP. Electrospun hydroxyapatite fibers from a simple sol-gel system. Mater Letters 2012;67:233-236.

31. Dubben S, Honscheid A, Winkler K, Rink L, Haase H. Cellular zinc homeostasis is a regulator in monocyte differentiation of HL-60 cells by 1 alpha\},25-dihydroxyvitamin D3. J Leukoc Biol 2010;87(5):833-844.

32. Soares-Schanoski A, Gomez-Pina V, del Fresno C, Rodriguez-Rojas A, Garcia F, Glaria A, Sanchez M, et al. 6-Methylprednisolone down-regulates IRAK-M in human and murine osteoclasts and boosts bone-resorbing activity: a putative mechanism for corticoid-induced osteoporosis. J Leukoc Biol 2007;82:700-709.

33. Kondo N, Tokunaga K, Ito T, Arai K, Amizuka N, Minqi L, Kitahara H, et al. High dose glucocorticoid hampers bone formation and resorption after bone marrow ablation in rat. Microsc Res Tech 2006;69:839-846. 
34. Xu JL, Khor KA, Sui JJ, Zhang JH, Chen WN. Protein expression profiles in osteoblasts in response to differentially shaped hydroxyapatite nanoparticles. Biomaterials 2009;30:53855391.

35. Alcaide M, Serrano MC, Pagani R, Sanchez-Salcedo S, Nieto A, Vallet-Regi M, Portoles MT. L929 fibroblast and Saos-2 osteoblast response to hydroxyapatite-betaTCP/agarose biomaterial. J Biomed Mater Res A ; 2009;89:539-549.

36. Alcaide M, Serrano M, Pagani R, Sánchez-Salcedo S, Vallet-Regí M, Portolés M. Biocompatibility markers for the study of interactions between osteoblasts and composite biomaterials. Biomaterials 2009;30:45-51.

37. Galluzzi L, Zamzami N, de La Motte Rouge T, Lemaire C, Brenner C, Kroemer G. Methods for the assessment of mitochondrial membrane permeabilization in apoptosis Apoptosis 2007;12:803-813.

38. Li X, Nan K, Shi S, Chen H. Preparation and characterization of nanohydroxyapatite/chitosan cross-linking composite membrane intended for tissue engineering. Int J Biol Macromol 2012;50:43-49.

39. Kailasanathan C, Selvakumar N, Naidu V. Structure and properties of titania reinforced nano-hydroxyapatite/gelatin bio-composites for bone graft materials. Ceram Int 2012;38:571579.

40. Zhang X, Cai Q, Liu H, Zhang S, Wei Y, Yang X, Lin Y, et al. Calcium ion release and osteoblastic behavior of gelatin/beta-tricalcium phosphate composite nanofibers fabricated by electrospinning. Mater Lett 2012;73:172-175.

41. Venugopal J, Low S, Choon AT, Sampath Kumar TS, Ramakrishna S. Mineralization of osteoblasts with electrospun collagen/hydroxyapatite nanofibers. J Mater Sci Mater Med 2008;19:2039-2046. 
42. Hilal Turkoglu S. Novel hybrid scaffolds for the cultivation of osteoblast cells. Int J Biol Macromol 2011;49:838-846. 